American Journal of Modern Physics
2015; 4(2-1): 9-14
Published online January 28, 2015 (http://www.sciencepublishinggroup.com/j/ajmp)
doi: 10.11648/j.ajmp.s.2015040201.12
ISSN: 2326-8867 (Print); ISSN: 2326-8891 (Online)

\title{
Time origin and universe uniform expanding
}

\author{
Michael H. Shulman \\ An independent researcher, Moscow ,Russia
}

Email address:

shulman@dol.ru

To cite this article:

Michael H. Shulman. Time Origin and Universe Uniform Expanding. American Journal of Modern Physics. Special Issue: Physics of Time: Theory and Experiment. Vol. 4, No. 2-1, 2015, pp. 9-14. doi: 10.11648/j.ajmp.s.2015040201.12

\begin{abstract}
A new cosmological hypothesis is considered which states that our Universe is a black hole in some external world. Because of that it expands linearly depending on so-called Parametric Time. For such the model we use the typical Einstein-Friedmann's equation system, but non-standard boundary conditions should be applied. A number of results are established that are indicative of the model efficacy.
\end{abstract}

Keywords: Standard Cosmological Model, Alternative Cosmological Model, Einstein's Equations, Black Hole, Relic Photons, Universe Entropy, Extremely Large Dirac Numbers, Universe Lifetime

\section{Introduction}

It is well known that the Standard Cosmological Model (or $\Lambda \mathrm{CDM}$ ) contains a number of discrepancies with the observed data and is criticized in the scientific literature. Particularly, recently several cosmologists departing from different reasons are proposed [1-6] independently the elements of a new cosmological concept, in which by contrast with $\Lambda \mathrm{CDM}$ the expanding Universe age is strongly proportional to its current size. Each of these publications contains the results of calculations that well correspond to the observed data. However, there is no there any fundamental explanation of such a model (excluding [2, 3]).

Since 1993 I also develop such the model [7-11]. This work was motivated by the attempt to introduce Time into the science as physically comprehensive quantity, not as a formal parameter. It was naturally to associate the universal (and irreversible) Time course phenomenon with the most general process in our Universe: its expanding. On the next step I simply identified the Universe current age with its current curvature radius.

However, this new concept's adepts do not point out the following fundamental issue: the linear link between the Universe age and size immediately leads to the contradiction in any cosmological model, in which the conservation energy law is held. In fact, the commonly accepted Universe evolution curve is deduced from such the law (see, e.g., [15]). It turns out that the strong proportionality between the Universe size and age immediately leads to the linear increasing its mass and energy with Time ${ }^{1}$.

The modern cosmology de-facto considers the Universe as a thermodynamically isolated system, and it supposes that its total energy and matter amount does not change over all its evolution time. However, Lee Smolin refers to J. A. Wheeler and wrote in [16]:

"It may then be conjectured that each black hole of our universe leads to such a creation of a new universe and that, correspondingly, the big bang in our past is the result of the formation of a black hole in another universe."

Can our Universe be a black hole? The correct answer is: our Universe can't not be a black hole in some external world. This statement proof is very simple. Let us consider an infinite universe having a given (average) density $\rho$ y and infinite mass. Furthermore, let us select a virtual sphere having a small radius $R$. If we will increase the virtual sphere radius, its mass $M$ will increase (as well as its gravitational radius $R_{G}$ ) proportionally to the cube of the geometrical sphere radius. In other words, the geometrical radius $R$ is proportional to the cube root from the mass $M$ and (hence) from the gravitational radius $R_{G}$. The non-linearity of this dependence means that starting from some critical value (depending on the density $\rho$ ) the gravitational radius will necessary overcome the geometrical sphere size; hence, this

\footnotetext{
1 The German physician (not a physicist!) Robert Mayer was the discoverer of the energy conservation law. He formulated his idea in the paper that he sent to J. Ch. Poggendorf's "Annalen der Physik". However, the paper was not published, and Poggendorf saved his "reputation" forever. It is interesting, what the fate waits now a publication (and its editor) that supposes our Universe to not to be a thermodynamically isolated system, so its total energy may to not be constant.
} 
spherical mass will become a black hole for which the critical density $\rho_{c r}=3 /\left(8 \pi R_{G}{ }^{2}\right)$ will be equal to the given density $\rho$. So, the gravitational collapse will be inevitable, because of that our real Universe cannot be infinite.

Let us now consider our Universe having the average density near to $10^{-29} \mathrm{~g} / \mathrm{sm}^{3}$. The calculation results of the parameter $\left(\rho / \rho_{c r}\right)$ showing the remoteness from the collapse state for different astrophysical objects are represented in the Table 1.

Table 1. Ratio $\left(\rho / \rho_{c r}\right)$ for different astrophysical objects

\begin{tabular}{lllll}
\hline Object & $\begin{array}{l}\text { Mass M } \\
(\mathbf{k g})\end{array}$ & $\begin{array}{l}\text { Radius } \\
\mathbf{R}(\mathbf{m})\end{array}$ & $\begin{array}{l}\text { Gravitational } \\
\text { radius } \mathbf{R}_{\mathbf{G}}(\mathbf{m})\end{array}$ & $\begin{array}{l}\left(\boldsymbol{\rho} / \boldsymbol{\rho}_{c r}\right)= \\
\left(\mathbf{R}_{\mathbf{G}} / \mathbf{R}\right)^{\mathbf{3}}\end{array}$ \\
\hline Earth & $6 \cdot 10^{24}$ & $6 \cdot 10^{6}$ & $10^{-2}$ & $\sim 10^{-26}$ \\
Sun & $2 \cdot 10^{30}$ & $7 \cdot 10^{8}$ & $3 \cdot 10^{3}$ & $\sim 10^{-16}$ \\
Milky Way & $3 \cdot 10^{42}$ & $\sim 10^{19}$ & $\sim 10^{15}$ & $\sim 10^{-12}$ \\
Universe & $\sim 10^{53}$ & $\sim 10^{26}$ & $\sim 10^{26}$ & $\sim 1$ \\
\hline
\end{tabular}

From this Table it follows that the entire Universe in fact should be in the gravitational collapse state.

Note, since any black hole irreversibly increases its mass and event horizon surface area while "eating" the external energy and matter, it expands like our Universe.

Such the cosmological model provides many arguments that confirm it. Moreover, it successfully competes with the Standard Cosmological Model, as I believe. I will shortly call this model as SEUT (Spherical Expanding Universe Theory).

\section{Possible Geometry of Black Hole}

As it is well known, for an external observer in our Universe a black hole $(\mathrm{BH})$ can be exactly represented by $2 \mathrm{D}$ membrane model that is located on the BH's bound. But what happens inside of $\mathrm{BH}$ ?

The common approach provides the "prolongation" of the internal solution into internal region of BH. As result, several exotic features appear including internal singularities. Further, the internal solution effectively depends on a given point location relative to the BH's center, and this fact contradicts to the observable Universe homogeneity. Hence, the solution prolongation idea is not consistent with our hypothesis.

Meanwhile, there exist different approaches to describe the $\mathrm{BH}$ interior region. Thus, the authors of the work [17] refused such a concept of the BH's internal structure and proposed the new solution for a body endpoint of gravitational collapse. By extending the concept of BoseEinstein condensation to gravitational systems they constructed a cold, compact object with an interior de Sitter condensate phase and an exterior Schwarzschild geometry of arbitrary total mass. These areas are separated by a phase boundary with a small but finite thickness (near to the Planck's length) of a fluid replacing both the Schwarzschild and de Sitter classical horizons. The new solution has no singularities, no event horizons, and has a global time. Its entropy is maximized under small fluctuations and is given by the standard hydrodynamic entropy of the thin shell. Unlike black holes, a collapsed star of this kind is thermodynamically stable.
On the other hand, my own study [18] basing on the General Relativity known results revealed a very interesting picture of that happens near to the finite size body gravitational collapse. Far from the collapse state pressure is positive and decreases continuously from the center of the body to its bound. However, it turned out that during the object contraction (but before the collapse event) a new situation appears: The pressure distribution inside of the object is fully changing. An infinite bipolar pressure break point in the center appears which is forced out to the bound while the collapse is approaching.

This impelled me to propose the more radical concept of description BHs in our Universe that also can be used as base to explain the Universe's features. The concept suggests that the membrane-shell really appears at the BH's event horizon, however, the space-time topology change happens there as a gravitational collapse result, and physical space itself disappears as such inside of $\mathrm{BH}$, the bound between the interior and exterior regions of 3Dspace has the dimension number 2. Then the representation like $2 \mathrm{D}$ membrane becomes to be absolutely exact, not approximate. The $\mathrm{BH}$ total mass turns out to be concentrated uniformly in this $2 \mathrm{D}$ region ${ }^{2}$, and there is no some difference depending on the distance from the BH's center ${ }^{3}$.

In my opinion, the BH's structure transforms at the collapse. There will be nothing inside of the object bound, all the matter will concentrate in the boundary shell, and the BH's dimension number reduces (new dimension number is old dimension number minus 1). Furthermore, the event horizon surface area increases while it consumes a matter and energy. From the hypothetical 2D observer point view who is disposed on the surface, its 2D universe increases and the real measure of the universe variability is its total mass value.

Note, for such an observer the energy conservation law will not accomplish in its universe, this energy will irreversible increase. Let us emphasize the following: $\mathrm{BH}$ consumes an external matter and increases its size like a living organism, such the behavior is similar to the biological metabolism process. For such the systems A. Levich introduced (see $[19,20]$ ) the notion of Parametric Time that simply is linearly proportional to a basic system resource (in this case - to the mass of the system).

\section{Our Universe as BH in an External World. The Formalism of SEUT}

When we compare our Universe's behavior with this situation, before all we find out that it expands too. In 1993 I reflected on the Kozyrev's ideas [21] and came to the Universe concept as a 3D shell of a 4D Euclidean sphere ${ }^{4}$

\footnotetext{
${ }^{2}$ Last time a number of publications appeared (including the paper of the such known author as V. Frolov), in which a close model was described [35, 36, 37].

${ }^{3}$ Now one may understand why the environment average entropy is proportional to the medium element volume, and the membrane entropy is proportional to its surface area element.

${ }^{4}$ The pseudo-Euclidean metrics appears in the model as artifact while one
} 
(see [7 - 14]). The increasing sphere radius I identified with the Universe age, so it received a simple and clear meaning of Parametric Time. In such a model the velocity of light has a status of an empirical coefficient to transit from length measuring along 3D sphere surface to the length measure along the normal to this sphere.

On the other hand, the velocity of light status as a maximally possible one simply corresponds with the maximal angle $(\pi / 2)$ of a possible inclination of a $4 \mathrm{D}$ world line relative to the spatial 3D sphere surface. Such a model can be deduced from the suggestion that our Universe is $3 D$ Black Hole, i.e., 3D membrane in a 4D surrounding environment.

We can write for such the Universe the usual EinsteinFriedmann's equations:

$$
\begin{gathered}
k(c / R)^{2}+(\dot{R} / R)^{2}+2(\ddot{R} / R)=-8 \pi G P / c^{2} \\
k(c / R)^{2}+(\dot{R} / R)^{2}=8 \pi G \rho / 3
\end{gathered}
$$

where $R$ is a curvature radius, $G$ is the constant in the Newton Gravity law, $c$ is velocity of light, $\rho$ is a matter density, $P$ is a matter pressure, $k=0,1$ or -1 depending on a curvature sign. Here $\dot{R}$ and $\ddot{R}$ denote the first and second derivative on time respectively.

In order to solve this system the cosmologists so far made the following assumptions. Firstly, Time was believed to be independent variable, and one did not limite a priori a dependence the curvature radius $R$ on Time. Secondly, one assumes total mass (and energy) in the Universe to be constant, independent on Time. Thirdly, one assumes the matter pressure (not the radiation one) to be zero ("galaxy dust" hypothesis). The last assumption forced out to introduce in this equations the non-zero cosmological constant $\Lambda$ in order to satisfy the observed data.

In the proposed model we go by another way. Contrary to the above described approach we explicitly introduce the Parametric Time that is exactly proportional to the Universe total mass. As it is well known, for a Schwarzschild's BH its mass is proportional to the (gravitational) radius. Because of that we set for Parametric Time $t=R / c$. In our model $R$ is the radius of an expanding 4D sphere. At each Parametric Time point $t$ our spatial Universe is represented by a closed 3D hyperspherical uniform surface ${ }^{5}$. Thus, we use the conditions $\dot{R}=c$ and $\ddot{R}=0$ while solving the Einstein-Friedmann's equations. Here $c$ is simply an empyrical factor connecting the length intervals that are parallel and normal to the $3 \mathrm{D}$ surface. Parametric Time axis is always oriented normally to this hypersurface.

Further, if we believe the Universe to be a $\mathrm{BH}$, its total energy (and mass) cannot be unchangeable. Generally, the energy conservation law use for expanding Universe leads to some contradiction because the global energy conservation is due to the Noether's theorem and its premise that Time is

interprets the mechanical motion meaning, see [7-10].

${ }^{5}$ From point of view of an "external" observer, a time interval when enrgy and matter are not coming from outside is similar to a single Parametric Time moment because any Universe evolution activity during this interval is "frozen". uniform. Meanwhile, in the early Universe the metric tensor component values (hence, gravitational force values and other physical quantities) were very different. In other words, Time in the expanding Universe cannot be physically uniform.

Finally, the physicists following the Einstein's tradition believed the matter pressure to be equal to zero. But such a statement was not due to a principle, contrary, it was artificial For example, in his classical monograph [22] R. Tolman describes the solution of the pressure and density distribution problem inside of a material sphere where he uses the nonzeroth pressure. Unfortunately, when Einstein considered the problem for his initial model of the static Universe, he did not find out a solution with positive pressure and was confused by this fact. Instead of the negative pressure he introduced its surrogate - cosmological constant $\Lambda$. This changes nothing in mathematics or physical meaning, however, confused the cosmologists.

Because of that in our model we do not limit a priori the energy and pressure dependence on Time, we search for them while solving the equation system. For the expanding Universe we naturally come to the energy evolution law insead of the conservation one. This law turns out to be linear as should be due to Parametric Time definition. The pressure turns out to be essentially negative, and this has a deep physical meaning (like the Einstein's static Universe model): the negative pressure just describes the mutual matter attraction, i.e., negative energy of Gravity.

Using the conditions $\dot{R}=\mathrm{c}, \ddot{R}=0$ we come to the new cosmological solution for the matter density $\rho$ and matter pressure $P$ on the curvature radius $R$ (that is proportional to Parametric Time):

$$
\begin{aligned}
& \rho=3 c^{2} /\left(4 \pi G R^{2}\right) \\
& P=-c^{4} /\left(4 \pi G R^{2}\right)
\end{aligned}
$$

So, the state equation has the typical form:

$$
P=-\rho c^{2} / 3
$$

As it should be, the total Universe mass is proportional to $R$ and t. Hence, the Kozyrev's prediction that "Time transforms to Energy" (see [21]) surprisingly turns out to be true. However, in modern epoch the relative level of energy non-conservation (per year) is near to $10^{-10}$, and it is very difficult to reveal it in a lab. But this effect may play a role in star and galaxy phenomena. In fact, the Sun's mass increment per year is few orders more than its loss to radiate.

So, with our approach the matter density and pressure are depending on the space curvature (are not introduced "by hand"), this fully corresponds to the Einstein's approach directed to geometrize the physics. It means (in the physics language) that matter pressure and density are features of the space curvature given to us "in sensations" (measurements). So, they represent secondary quantities, i.e., depending on it. Let us remark, such the way was denoted by Einstein himself while he introduced the Universe closed onto it-self. Thus, he 
replaced fixed boundary conditions by the condition of selfconsistency!

\section{Discussion}

The detailed description of the model and its results is given in my publications (see reference list at the end of the paper). Here we shortly discuss the key model statements.

4.1. Before all, the physical meaning of Big Bang is specified. This is our Universe creation act as an object gravitational collapse in some External World. Because of above arguments such the Universe does not contain any singularities.

4.2. The maximal velocity existence can be connected with the maximal inclination angle $(\pi / 2)$ between a moving particle word line and the 3D hypersphere curvature radius. A gravitation force is also interpreted geometrically as an inclination angle between its direction and the same radius, because of that kinetic energy and gravity potential one can mutually be transformed one to another.

4.3. In the SEUT the Universe radius and the event horizon rise proportionally due to linear evolution. This resolves the known "horizon problem", one does not need in the "initial inflation" hypothesis.

4.4. The nova-day observation allowed us to find out the angular size $\sim 0.6^{\circ}$ corresponding to the maximal peak of the CMBR spectrum [23]. From that one deduces in the Standard Cosmological Model (SCM) that the Universe spatial geometry is flat. Further, from it follows that average matter density is practically equal to the critical one. If one uses the especially fitted value of $\Lambda$, then he reveals in SCM a nonlinear $R$ dependence on Time. From this one concudes that the Universe expands with some acceleration in the modern epoch, so our epoch seems to be a special one.

Meanwhile, in the work [24] it is shown that exact location of the spectrum maximal peak can be determined independently on the Universe spatial metrics type. At this our model states:

- Our Universe metrics at any evolution time point has a positive curvature and (respectively) spherical geometry, its real density is always two times more than critical one ${ }^{6}$.

- The Universe over all the evolution time expands with a constant rate, and our epoch is not an especial one, so there is no any accelerating (or decelerating) expansion.

4.5. The hardly established phenomenon of the CMBR dipole anisotropy is in some collision with the fundamental idea of the Relativity on the preferred reference frame absence. But our model (SEUT) just supposes that there is such a preferred reference frame at every spatial point of the Universe that explains the dipole anisotropy phenomenon [25]. However, the velocity corresponding to this anisotropy is only $\sim 0.001$ of velocity of light, because of that we have a good concordance with relativistic picture.

${ }^{6}$ This is confirmed by the observed data connecting galaxies angular size with its redshift (see [30]).
4.6. There is one more interesting aspect. The both CMBR temperature power spectrum and temperature-polarization cross-spectrum have the peak ${ }^{7}$ at the multipole number $\ell \approx 5$. The SCM is not able to explain satisfactory this phenomenon. However, our model predicts the existence of just such the peak due to relic photons travelling along the expanding Universe over $(360+40)^{\circ}$, see [26].

4.7. As it is known, the forced introduction of the non-zero $\Lambda$ in the SCM creates a new (practically unresolved) "problem of the vacuum" (see review [27]): The estimation of the vacuum energy is 122 order less than quantum mechanical calculations actually give. Furthermore, in my opinion, the vacuum zero-point oscillations energy cannot be extracted and used for the Universe gravitational expansion, nor for any something, because it corresponds to the lowest energy possible state. Finally, the Universe size changes with time, while the value of $\Lambda$ is considered as constant. What about the SEUT, it does not contain the cosmological constant, however there is the same concordance with the observed data as in the SCM.

4.8. In the SCM the fact that at given redshift a distant Supernova seems to be dimmer than one expected is explained using the especially fitted cosmological constant value $\Lambda$. Meanwhile, in the SEUT one does not need use some "free" parameter $\Lambda$, it gives immediately the result that corresponds to the observed data and the SCM prediction [28].

4.9. There is the important cosmological test: the mean galaxy angular size dependence on redshift. Several recently published papers show that the observed data does not correspond with the SCM prediction. Meanwhile, we made some theoretical investigation where practically obtained the SEUT predictions satisfactory concordance with the observed data using certain assumptions (see [29]).

4.10. The present-day cosmology de-facto considers the Universe as thermodynamically closed system, particularly while one integrates the Einstein-Friedman equations. This generates a number of difficulties when one explains the actual situation including the total discrepancy from the equilibrium state. Because of that de-jure the cosmology refers the General Relativity that considers the world as a system in the alternative gravitational field (not as closed system) for which the second law of thermodynamics can not be satisfied. My model proposes a new point of view on our Universe thermodynamics. In this model the Universe entropy decreases (not increases) since (like working medium of a heat engine) it receives energy from outside at a relatively high temperature (few kelvins) and gives it up to own (interior) supermassive BHs at a practically equal to zero temperature ${ }^{8}$. Because of that the cosmological Arrow of Time origins from thermodynamics and is primordial relative to biological (evolution) and psychological Arrows. This is the reason of a Universe structure continuous differentiation

\footnotetext{
See [31].

${ }^{8}$ The supermassive black holes in the centers of galaxies give a dominant contribution into our Universe's entropy (see [33]).
} 
and increasing deviation of the Universe state from equilibrium during 13.8 billions years of Parametric Time [32].

4.11. In the September of 2013 I revealed one more serious argument that has confirmed the SEUT (as I believe). It is associated with so-called "extremely large Dirac numbers". The full description of this problem is given in [34], and here I describe its resolution shortly. On one hand we can define the Plank mass and size using dimensionality reasons only:

$$
m_{p}=\sqrt{\frac{\hbar c}{G}} \approx 2.1 \cdot 10^{-5} \mathrm{~g}, l_{p}=\sqrt{\frac{\hbar G}{c^{3}}} \approx 1.6 \cdot 10^{-33} \mathrm{~cm}
$$

Note that their ratio is

$$
\frac{m_{p}}{l_{p}}=\sqrt{\frac{(\hbar c / G)}{\left(\hbar G / c^{3}\right)}}=\frac{c^{2}}{G} \approx 10^{28} \mathrm{~g} / \mathrm{cm}
$$

On the other hand, from the well known link between a body gravitational radius and mass $\left(R=2 G M / c^{2}\right)$ we can deduce the same ratio between the nowadays Universe corresponding parameters:

$$
\frac{M_{U}}{R_{U}} \approx \frac{c^{2}}{G}
$$

If $m_{p}$ and $l_{p}$ specify our Universe immediately after Big Bang, then one could see that this ratio remains always constant, so the SEUT may be considered as confirmed. One also can see that the important expression follows from the two preceding relationships:

$$
\frac{M_{U}}{m_{p}}=\frac{R_{U}}{l_{p}} \approx 10^{60} \equiv D
$$

This quantity that I proposed to name "Dirac's number" is dimensionless Universe lifetime.

\section{References}

[1] Shlomo Barak and Elia M Leibowitz. Cosmology and Astrophysics without Dark Energy and Dark Matter" (arXiv: astro-ph 0909.2581).

[2] A.Benoit-Lévy and Gabriel Chardin. Do we live in a DiracMilne Universe? (arXiv:0903.2446v1).

[3] A.Benoit-Lévy and Gabriel Chardin. Introducing the DiracMilne Universe. (arXiv:1110.3054v1)

[4] F.J.M. Farley. Does gravity operate between galaxies? Observational evidence re-examined (arXiv:1005.5052v1)

[5] Melia F., Shevchuk A. S. H., 2012, MNRAS, 419, 2579

[6] Melia F. The Cosmic Spacetime. Is The Universe Much Simpler Than we Thought? (arXiv: 1205.2713v1)

[7] M. H. Shulman. On Time physical origin. Moscow, IRC RAO "Gazprom", 1997 (in Russian).

[8] M. H. Shulman. Cosmology and Time Origin. In
"Freedmanovskie Chtenia", All-Russian Scientific Workshop, Perm, 7-12 September, 1998 (p.p. 20-22) (in Russian).

[9] M.H. Shulman. Time as Phenomenon of the Expanding Universe. New Energy Technologies. Issue \# 4 (7) JulyAugust 2002.

[10] M.H. Shulman: Paradoxes, Logics, and Physical Nature of Time. http://www.timeorigin21.narod.ru/rus time/Origin.pdf (in Russian) On the Time, Motion and Matter physical Meaning.

$\mathrm{http}$ //www.chronos.msu.ru/EREPORTS/shulman on the tim e.htm (in English)

[11] M.H. Shulman. Cosmology: a New Approach. http://www.timeorigin21.narod.ru/eng_time/Cosmology.pdf

[12] M.H. Shulman. Kozyrev's Time. In "Time and Stars: the Centenary of N.A. Kozyrev ", Nestor-Istoria, St. Petersburg, 2008 (p.p. 556-561) (in Russian).

[13] M.H. Shulman. Time as Phenomenon of the Expanding Universe. In "On the Way to Understanding the Time Phenomenon: the Constructions of Time in Natural Science". Progress-Traditia, Moscow, 2009 (p.p. 207-234) (in Russian)

[14] M.H. Shulman. Alternative Cosmology (in Russian). http://www.timeorigin21.narod.ru/rus_time/Alt_cosmology.pd $\mathrm{f}$ See short book's presentation in English http://www.timeorigin21.narod.ru/eng_time/Presentation_eng. pdf

[15] A. Einstein. The Meaning of Relativity. Princeton, 1953.

[16] Lee Smolin. The fate of black hole singularities and the parameters of the standard models of particle physics and cosmology. ArXiv:gr-qc/9404011v1 7 Apr 1994

[17] Pawel O. Mazur and Emil Mottola. Gravitational Condensate Stars. An Alternative to Black Holes. ArXiv:gr-qc/0109035v5 27 Feb 2002

[18] M.H.Shulman. Usual collapse and unusual one. http://www.timeorigin21.narod.ru/eng_time/Collapse.pdf

[19] A.P. Levich. Metabolic time of natural systems // System Studies. Annual edition. 1988. Moscow: Nauka. 1989. Pp.304325 (in Russian).

[20] A.P. Levich. Time as Variability of Natural Systems: Ways of Quantitative Description of Changes and Creation of Changes by Substantial Flows // On the Way to Understanding the Time Phenomenon: the Constructions of Time in Natural Science. Part 1. Interdisciplinary Time Studies. Moscow, Singapore, New Jersey, London, Hong Kong: World Scientific. 1995b. Pp.149-192. http://www.chronos.msu.ru/EREPORTS/levich1.pdf

[21] N.A. Kozyrev. Selected works. Leningrad, Edition of the Leningrad State University, 1991 (in Russian).

[22] R.C. Tolman. Relativity, Thermodynamics, and Cosmology. Oxford, Clarendon Press, 1934.

[23] Komatsu et al. Seven-Year Wilkinson Microwave Anisotropy Probe (WMAP) Observations: Cosmological Interpretation. ArXiv: 1001.4538v2 [astro-ph.CO] 12 Feb 2010

[24] M.H. Shulman. Universe expansion and main spectral peak of CMB. http://www.timeorigin21.narod.ru/eng_time/Main_peak_eng.p $\mathrm{df}$ 
[25] M.H. Shulman. On an experimental validation of the selected reference frame existence in the Universe. $\mathrm{http}: / /$ www.timeorigin21.narod.ru/eng time/On the selected reference_frame.pdf

[26] M.H.Shulman and G.Raffel. On the oldest photons phenomenon.

http://www.timeorigin21.narod.ru/eng_time/Oldest_photons_e ng.pdf

[27] Raphael Bousso. TASI Lectures on the Cosmological Constant. ArXiv: 0708.4231v2 [hep-th] 11 Sep 2007

[28] M.H.Shulman. On the supernovae low luminosity problem. http://www.timeorigin21.narod.ru/eng_time/On_supernovae.p df

[29] M.H. Shulman and G. Raffel. On galaxies angular size evolution.

http://www.timeorigin21.narod.ru/eng_time/angle_size_evolut ion_eng.pdf

[30] Weinberg S. Gravitation and Cosmology: Principles and applications of the General Theory of Relativity. John Wiley and Sons, Inc., 1972.

[31] Dunkley et al. Five-Year Wilkinson Microwave Anizotropy Probe (WMAP) Observations: Likelihoods and Parameters from the WMAP data. ArXiv: astro-ph/0803.0586v1 5 Mar 2008

[32] M.H. Shulman. Time, entropy, and Universe. $\mathrm{http}: / /$ www.timeorigin21.narod.ru/eng_time/Time_and_entrop y_eng.pdf

[33] Ch. Egan and Ch. Lineweaver: A larger estimate of the entropy of the universe. ArXiv: 0909.3983v1 [astro-ph.CO] 22 Sep 2009.

[34] "Extremely Large" Dirac Numbers and Fundamental Constants in Cosmology. http://www.timeorigin21.narod.ru//eng_time/Dirac_number_e ng.pdf

[35] Pawel O. Mazur and Emil Mottola. Gravitational Condensate Stars: An Alternative to Black Holes. ArXiv:gr-qc/0109035v5 27 Feb 2002

[36] Valeri P. Frolov. Do Black Holes Exist? arXiv:1411.6981v1 [hep-th] 25 Nov 2014

[37] Robie A. Hennigar and Robert B. Mann. Super-Entropic Black Holes. ArXiv:1411.4309v1 [hep-th] 16 Nov 2014 